"no patient should be put on the treatment until he had been from twenty-four to thirty-six hours in the wards, and then only when it seemed clear that his illness was running an acute course." His object in giving these instructions was to exclude such cases as would, "independently of any medicine, improve rapidly after admission into hospital and become convalescent in three or four days." His plan, however, was not always quite strictly carried out, and the consequence was that he dismissed as valueless no fewer than twelve of the cases in which the subsidence of symptoms under treatment was most rapid. But Dr. Fagge urged that really the very question at issue is whether salicylic acid is, or is not, capable of shortening the duration of the attack. He certainly knows of no evidence that so large a proportion of cases as this (in addition to an indefinite number of other cases withdrawn from treatment on the ground of their mildness) would ever get well in from one to four days without treatment. The figures given by Sir William Gull and Dr. Sutton (see Table I.) fail altogether to support such an opinion. He therefore thinks that Dr. Greenhow did really obtain good results with salicylic acid and salicin, but that in his anxiety to weigh strictly the therapeutical claims of these drugs he dealt them scant justice. That the severity of his sixty cases was much above the average seems to be shown by the fact that in about twenty-five of them pericarditis was discovered, either on admission or a day or two afterwards. The last two questions to be discussed were as regards the possible action of salicylic acid and salicin in preventing the development of the cardiac complications of acute rheumatism, and in warding off the risk of dangerous or fatal hyperpyrexia. As regards the heart one could not but remember that for each new method of treatment introduced within the last two years, eren for the treatment by local blistering, one claim invariably made had been that it lessened the liability to inflammation of that organ. On the other hand, Sir W. Gull and Dr. Sutton showed that in cases in which the heart is healthy at the time of the patient's admission into hospital it seldom becomes subsequently attacked. Still, it is only fair to expect that any remedy which possesses the power of arresting acute rheumatism, so that fresh joints no longer become affected, must also hinder the development of what is believed to be an analogous morbid process in and around the heart. And although in sixty-nine of the 355 cases auscultation revealed some change in the character of the heart-sounds while the patient was in the hospital, Dr. Fagge could hardly find one in which there was reason to believe that pericarditis set in at a time when the action of the remedy was fully established. Almost all of them were cases in which at one period or another a systolic murmur was detected; and he believes that considerable doubt often hangs about the interpretation of such sounds. On the other hand, he fully admits that salicylic acid seems to have no power of controlling or arresting the cardiac complications of acute rheumatism, when once they have developed themselves. As to hyperpyrexia, Dr. Fagge thinks that the evidence, so far as it goes, is favourable to the drug. It would seem, however, that the risk of this and other dangerous complications of acute rheumatism has been far greater in some years than others. Between 1855 and 1867 only ten fatal cases of acute rheumatism in all seem to have occurred at Guy's Hospital. Between 1868 and 1880 there were forty-seven cases, no fewer than thirty-six of which were in the years 1870-1877 inclusive. At that time Dr. Fagge formed an impression that the frequency of fatal hyperpyrexia was not altogether unconnected with the fact that quinine and the tincture of perchloride of iron-medicines which tend to check sweating-were very commonly administered to patients affected with acute rheumatism, and that it was the practice to wrap up the swollen joints in masses of cotton-wool. The tincture of iron was being given to two of the patients whose cases are recorded by Dr. Wilson Fox as having been treated by cold baths. There can, of course, be no question that the profuse sweating which accompanies this disease, whether useful or injurious to the patient in other respects, must greatly tend to keep down the pyrexia. Now in rheumatic hyperpyrexia the rule is that the skin becomes dry as the temperatnre rises, though to this rule there are some exceptions. It may, therefore, be important not to administer to rheumatic patients any medicine which, besides having antipyretic properties, also checks the function of the sweat-glands, lest the former action chancing to fail, the latter should increase the patient's risk. But no such objection would apply to salicylic acid, which rather causes sweating than tends to diminish it As a matter of fact, byperpyrexia occurred in two of the 355 cases included in Dr. Fagge's series, but one patient had taken the medicine (in twenty grain doses) for only twentyfour hours, when the temperature reached $1064^{\circ}$; and of the other patient it is not positively stated in the clinical report that he was raking the medicine at all when, five days after admission, the complication occurred. What would really show that salicylic acid is sometimes incapable of preventing the occurrence of hyperpyrexia would be that after the temperature had begun to fall under the administration of the remedy its course should change and that it should rapidly rise to a dangerous height. Recognising that the efficient dose of salicylic acid varies in different cases, and, therefore, that there may not improbably be some cases in which no dose that can be given is large enough to affect the disease, Dr. Fagge is quite prepared to believe that it may sometimes happen that the temperature remains high and rises to a fatal point in spite of the administration of the remedy. On the other hand, he could not refrain from remarking that Dr. Greenhow was hardly justified in speaking of two of his cases as having presented hyperpyrexia under treatment by salicylic acid, for in one of them the patient had been taking only fifteen grains of the salicylate of soda at intervals of six hours, and even this was discontinued the night before the temperature began to rise to an alarming height. In the other case the thermometer indicated $105.8^{\circ}$ at a time when twenty grain doses of the salicylate was being taken; but the progress upwards was by no means rapid, and no cerebral symptoms were manifested; it is therefore quite possible that a fall might afterwards have taken place if the treatment had not been interrupted. In conclusion, Dr. Fagge remarked that his hearers would, of course, see that he was a decided advocate for the administration of salicylic acid and salicin, and that, before he had made the investigations, of which the results were before them, he was strongly biased in favour of these medicines. He could, however, honestly declare that this opinion had been forced upon him by his clinical experience and that if his figures had now suggested a doubt to his mind he would have candidly and frankly admitted it. Indeed, when he first made trial of the drugs he was altogether sceptical as to their value; and even after a few patients had rapidly recovered he remembered cautioning his clinical clerks not to attach much importance to this, for he had sometimes seen the administration of other medicines followed by results that had appeared equally striking. But when case after case occurred with scarcely a failure, he became satisfied that he had a most potent remedy in his hands. All further experience had strengthened this conviction in his mind, and he would now feel that he was accepting a very grave responsibility if he were to withhold a drug which he believed to be so useful from any patient placed under his care, unless there were some good reason for doing so.

ON THE

\section{USE OF CHIAN TURPENTINE IN CANCER.} BY PROFESSOR JOHN CLAY,

OBSTETRIC SURGEON TO THE QUEEN'S HOSPITAL, BIRMINGHAM.

MoRe than two years have elapsed since I commenced treating cancer of the female generative organs with Chian turpentine. By the courtesy of the conductors of THE LANCET the results of $\mathrm{my}$ first experiments were pablished in the number for March 2/th, 1880. The paper attracted considerable attention, both professional and general, and provoked much adverse criticism ; indeed, in certain quarters this plan of treating cancer of the uterus in particular was declared useless. An enlarged experience, however, has confirmed the statements made in my original paper, and I have now the satisfaction of being able to declare that I have nothing to withdraw or to qualify as regards the statements I then made, as the result of observation, as to the effects of Chian turpentine in uterine cancer. I should be glad to confirm these conclusions by now publishing a number of illustrative cases showing the treatment pursued in different 
forms of uterine cancer; but consideration of the space at my disposal obliges me to be content with describing briefly the conditions under which a measure of success may be obtained, and the opprobrium of the alleged uselessness of the remedy may thus be removed, to the ultimate benefit of suffering humanity. It appears therefore necessary to determine whether Chian turpentine dies actually alleviate the distress of cancer, or has any contrulling influence upon the progress of the disease, or any pretensious 10 effect its cure. It is obvious that if either or all of these results can be secured by the use of the drug, in any form or situation of cancer, the remedy cannot be deemed useless. Now, the facts within my own knowledge, derived from my own professional experience, may be summed up as follows. Nine cases of eancer confined to the uterus, which have been under treatment for about twelve months, are so far convalescent that they are no longer under observation. The cancerous growths have disappeared, there is no bleeding on manipulation, and the parts are smooth to the touch, and appear to be covered with mucous membrane. In most of the cases the cervix uteri is shortened from the contraction consequent on the removal of the growth. A number of cases of uterine cancer in private and hospital practice are under treatment in which freedom from pain, diminution of hæmorrhage, and sloughing of the growth, with improvement of the general health, are prominent features. A number of advanced cuses of uterine cancer have been treated for a short time, and the patients have died, but an amelioration of the more severe symptoms took place, although the patients succumbed to the anæmia produced by the previous exhausting discharges. The disappearance of the cancerous growth was verified in three cases where an examination was made after death. One case was complicated with cystic disease of each ovary, one died from dysentery, and in the other the glands in the pelvis and abdomen were extensively diseased. In neither of the fatal cases where the remedy had been exhibited for some time were there any fistulous communications with the rectum or bladder. If we bear in mind the progressive and generally rapid advance of the disease when left alone-as it rarely disappears spontaneously - or when it is treated by palliatives, and compare this with the treatment by Chian turpentine, we find in the latter method a gradual subsidence of the disease, varying as to length of time in proportion to the more solid consistence of the growth, a marked diminution of pain, a lessening of the hæmorrhage, with an increase of the muco purulent discharge. There is usually an improvement in the general health, but an increased tendency to anæmia is sometimes noticed. From a review of all the cases I have observed, it may be safely asserted that the effects of Chian turpentine in cancer of the uterns are tolerably uniform, and it is more than probable that the remedy effectually removes the cancerous infiltration surrounding the original growth, thereby preventing the extension of the disease. In some instances the treatment has removed glandular complications, and in others it is presumable that these have been averted. It is this controlling action of the drug which probably causes the abatement of the pain and hemorrhage. The growth gradually diminishes in size, becoming loose and shrivelled, and losing its firm and succulent condition. Whatever may be the termination of the case, the treatment manifestly affords to the patient such comfort as is not obtainable by any other therapeutical measure.

The earlier the cancerous disease comes under treatment, the greater is the prospect of ultimate relief. The success obtained in recent cases is probably owing to the patients coming under observation before any apparent extension of the disease, or before vital organs are involved. Where the vagina is affected with the disease primarily or secondarily, particularly the latter, the prospects of relief are materially reduced. The treatment should be prosecuted vigorously and persistently, especially at the outset, so as to minimise the constitutional effects of the disease, as it is difficult to decide in what stage these are developed, and perseverance with the treatment should be strenuously encouraged by the medical attendant, as otherwise the patient may be left to die unaided by the only drug that has been found by its internal administration to have any pretensions to resolve a cancerous growth.

In treating a case of cancer of the uterus or rectum, the following procedure is recommended for adoption. It may be premised that it is essential that the genuine drug only should be administered. It is a humiliating statement to make that even now some houses are supplying and dis. pensing other turpentines for Chian turpentine, and are even guaranteeing the genuineness of the article they supply. It is also necessary that the drug should be given in the form which is most convenient for assimilation. The essence of Chian turpentine, prepared by Messrs. Southall and Barclay of Birmingham, appears to me to be the most suitable preparation. This is prepared without the sulphur, and evidently does not contain any ether. The drug is in a state of minute subdivision, is easily digestible, suitable for all forms and situations of cancer, and is very palatable. One teaspoonful of the essence contains three grains of the turpentine. In place of the sulphur in the essence, Messre. Sonthall prepare pills which contain sulphur, sulphate of copper, \&c., and which they style, "pil. sulph. comp.," to distinguis $\mathrm{h}$ them from the Chian turpentine pills. Two teaspoonfuls of the essence, with one or two of the compound sulphur pills, should be given three or four times a day, and after the medicine has been taken for about three montbs it should be omitted for about three days in every fortnight The pills or mixture, prepared according to the original formula, may be given instead of the essence and sulphur pills.

The vagina and rectum, even from the first, should be syringed daily with equal parts of vinegar and water. After allowing time for this to drain from the parts, it is advisable to insufflate into the vagina or rectum about ten grains of the following powder :- Tannic acid half an ounce, powdered charcoal two drachms, and powdered sulphate of copper ten grains. Messrs. Mappin and Co. of New-street, Birming. ham, make a vaginal insufflator by which the powder may be introduced into the rectum or vagina very efficiently. An insufflator may be improvised out of a vulcanite tube and a two-ounce india-rubber bottle connected with elastic tubing, but such an instrument is apt to become moistened by the secretions, and thus prevent the powder from becoming properly placed. The propriety of excising the os uteri in epithelioma of this part, as a preliminary procedure to the use of the Chian turpentine, may be questioned on reasonable grounds. The cancerous growth as it disappears under the Chian turpentine treatment leaves a shortening of the os uterı which brings the external rim of the os uteri into close proximity with the bladder and rectum, so that if the vaginal portion of the uterus has been removed the subsequent contraction of the lower portion of the uterus drags on the rectum and bladder, causing great pain, with rectal and especially vesical troubles. In large pedunculated epithelioma of the os uteri the larger portion of it is perhaps best removed, taking care to leave the normal nterine tissue intact. In cases where the turpentine has been taken for some months, the dull curette may be used to remove the sloughing mass with advantage. When pain in the sacral or hypogastric regions comes on after the treatment has been pursued for two or three months, the use of morphia suppositories is indicated. To avoid the habitual use of opium, the tincture of Jamaica dog. wood (Christy's), in one-drachm doses once or twice during the evening, may be prescribed. Hæmorrhage at the monthly periods is best met by giving the liquid extract of ergot in ten- or tifteen-minim doses (which may be added to the essence of Chian turpentine), and to use locally the perchloride of iron in solution, or the dried persulphate of iron by means of insufflation. Diarrhoea and dysentery sometimes supervene during the treatment, for which the oil of eucalyptus globulus (in tive-minim doses three times daily for a few days only) is a good remedy. This drug may be added to the essence of Chian turpentine. Anæmia consequent upon the occasional hæmorrhages and serous dis. charges is a serious complication; much of the local treatment here recommended has been suggested to anticipate or prevent the anæmia. To combat this condition, Fellows' syrup of the hypophosphites, in one-drachm doses, may be given during meals, from the commencement of the treat. ment, in counexion with the Chian turpentine treatment.

In cancer of the vulva Chian turpentine acts slowly, and as the disease frequently extends rapidly, an early excision of the growth, if possible, is necessary, the remedy being given for some time afterwards with a view to prevent a recurrence of the disease.

The use of Chian turpentine in a large number of cases of cancer of the breast shows that it is a remedy of considerable power in relieving pain, of diminishing the size of the growth by causing the removal of the cancerous infiltration, leaving the more permanent fibrous stroma to be subse- 
quently dealt with. In many instances the growth, although at first it was firm, and seemingly adherent to the chest walls, has become loose and easily movable from side to side, as if it were merely a foreign body, so that if an incision had been made in the skin it could have been pushed out. Several cases of primary scirrhus of the breast have been noticer in which, after the administration of the Chian turpentine for some months, the whole of the cancerous mass has sloughed away, appearing as a black gangrenous mass, leaving a cavity of considerable dimensions, the walls of which were composed of healthy tissue, and the wound healed with comparative rapidity by granulation. This process, however, is a slow and painful one, and suggests the propriety of anticipating the sloughing process by removing the growth with the knife after it has become sufficiently detached from the surrounding structures by the treatment. Several cases treated by this method have done well, the operation has been materially simplified, and, if the remedy is administered for some time after the operation, the experience gained shows that there is not so much likelihood of a recurrence of the disease. In recurrent cancer of the breast the remedy is often found to be of great benefit, and after it has been given for some weeks the application of the crystals of resorcin to the growth facilitates the disintegration of the mass. Resorcin is a powerful and at first a painless application. When its use is attended by much pain it may be mixed with equal portions of tannic acid and charcoal with good effect. Vaseline or chrisma dressing is all that is required after the application of the powder.

In epithelioma of the face and other parts of the cutaneous surface the use of Chian turpentine in conjunction with the powder previously advised (when stating the local treatment of cancer of the uterus), to which three drachms of resorcin have been added, very good results have been obtained. In two cases of cancer of the stomach very beneficial results have accrued from the special treatment. In cancer of the mouth and tongue the results have not been so good, in consequence of the rapidity with which the neigh. bouring glands became involved in the cancerous disease.

I leave these necessarily incomplete observations to the impartial critical judgment of the profession. Enough, I trust, has been stated to show that the Chian turpentine treatment has some ameliorating influence on cancerous disease--how much the future must determine, - and therefore I am enabled to claim that the remedy is far from being useless. The facts stated may be considered at least as a distinct addition to our existing stock of knowledge in the treatment of cancer, and I look forward as the almost certain result to their being confirmed, and to candid inquiry leading to further improvements in the same direction.

\section{ON SEA-SICKNESS.}

\section{BY JAMES REGINALD STOCKER, M.B.}

THERE are some diseases, the specific fevers for example, for which there is no cure, nor is it in the course of nature that there should be. But the system, having once encountered them, is, generally speaking, no longer liable to their attacks. The only security against such diseases is either to keep well out of their way, or by submitting to them to undergo what may be called a personal experience of them. There are also, it cannot be denied, certain conditions of the body which render it more or less susceptible to the influence of the poisons that engender them. They depend upon age, sex, condition; occupation, temperament, habits, and the like. These conditions, when favourable, appear rather to establish a kind of tolerance than to confer complete im. munity. When we look around us we cannot fail to perceive that some individuals appear at times to possess this property ; their systems are either acclimatised or accustomed, or from other causes are not susceptible, to them; at any rate they are free.

We observe a similar kind of tolerance sometimes, indeed generally, acquired by the system with regard to the special senses, particularly in certain occupations. So it is with regard to sea-sickness. Can we wonder that in this case too the system should sometimes become inured or accustomed to the sensations that produce it, and no longer amenable to their influence? Can we be surprised that in certain cases, or at certain times, men should seem to be endowed with the faculty of accommodating themselves to the singular sensasions that occur on board ship?

Having said so much by way of preface to my remarks, I may now proceed to discuss the nature or the pathology of sea-sickness. The pneumogastric nerve is always an interesting nerve to study. In the present inquiry it is an important one ; it is the key to the explanation of sea-sickness. It is the principal nerve of organic life ; it governs the heart, the lungs, and the stomach and intestines, as well as other more remote but yet important parts. It may seem curious, but it is nevertheless a fact, that the stomach sympathises most with the senses - the heart and lungs with the emotions, the ideas, and the intellect. If a powerful stimulus occurs to the mind, it affects principally the heart and lungs: if to the senses, the stomach is affected. The same may be said of sedatives or soothing influences.

The pneumogastric nerve sympathises, then, with the senses and the intellect, and plays an active part in that expression of disgust which results in vomiting. We see this thing occur as a consequence of an unpleasant impression upon any one of the special senses-the sense of taste, of smell, sight, hearing, and touch. Stimulus of the nerve, in moderation, favours digestion and the various other processes of organic life ; in excess it irritates them ; it directly occasions nausea, dyspepsia, flatulence, vomiting, \&c., and, indirectly, all the other sad effects of sea-sickness. The nervous centres, excited by the sensory impressions, become at last so irritable that the introduction of anything into the stomach, either from within or from without, is resented, and vomiting occurs; until sooner or later the nervous system is dominated by that potent influence for good or evil, the force of habit, and the body finally becomes accustomed to the $n \in W$ sensation.

I have spoken of a difference in the degree of stimulus: quantity. I will now allude to a difference in the kind: quality. There are some sensations that are pleasant and agreeable; others that are painful and disagreeable. It is not by any means always the case that the same sensations produce the same effect-i.e., of pleasure or of pain-either in the same or different persons, "what is one man's food is another man's poison." In other words, tastes differ; not only in different persons, but also at different times. An odour-e.g., as of cooking, may be grateful at one time, ungrateful at another; a sound agreeable at one time, at another disagreeable. 'It is peculiarly so with regard to movement. One is sometimes pleasantly affected by a motion which at other times may be quite the reverse, and it always bas a different effect upon some people from what it has upon others. I once met a man who said he had crossed the Atlantic eight times as a bachelor without being sea-sick; the ninth time he was married and had his wife with him ; he was sick the whole way.

The consideration of the phenomena of sea-sickness has convinced me that the fifth sense, commonly called the "sense of touch" or "common sensation," is a compound sense. By its means we are able to recognise not only touch and its varieties, but also distance, form, size, weight, consistence, relation, and time, and sometimes even colour and sound. "The capacity, therefore, of the hand," says Sir Charles Bell, " to ascertain the distance, size, weight, form, hardness or softness, roughness or smoothness of objects, results from its having a compound function-from the sensibility of the proper organ of touch being combined with the consciousness of the motion of the arm, hand, and fingers."-(Bell "On the Hand," 8th ed., London, 1877, p. 156.)

Nor is feeling by any means the only sense that is compound in its character, for all the other senses are more or less of a complex nature. Bell, indeed, may have been mistaken in his views as to the "muscular sense" being a sense apart and distinct from the others, for it doubtless properly belongs to the various special senses with which the muscles concermed are connected, the muscular apparatus of the trunk and limbs enabling and assisting them to fulfil their functions just as much as the muscular appendarges of the eye, the ear, the nose, and the tongue, mimister to them.

This, then, is one of the lessons that the study of sea-sickness teaches us. It teaches us an important physiological fact, that there is in us a sense which, without some such experience, we might perhaps be slow to recognise-the sense of passive motion. It may not indeed be so exalted a sense as others, nor so important, but it is certain? one 